\title{
The study of wind effects on the bridge constructions
}

\author{
Olga Poddaeva , Anastasia Fedosova and Julia Gribach* \\ Moscow State University of Civil Engineering, 26, Yaroslavskoe Shosse, 129337 Moscow, Russia
}

\begin{abstract}
Construction of bridge structures occupies one of the leading positions in the construction industry. However, the design of these objects is a complex multi-factor task. Particular attention in the development of bridge structures must be paid to wind loads, since their dynamic loads can cause a number of adverse aeroelastic reactions. Large-scale bridge structures, which are flexible structures, are more susceptible to horizontal influence of the wind. This article presents a description of the study of wind impact on a bridge being built in the city of Moscow, according to a technique that was developed by employees of the UNPL AAISK (NRU MGSU). In this case, a test of the span section of the model on a specialized stand was conducted. The example of this object shows a graph of the dependence of the amplitude of oscillations of the bridge span on the wind speed without the use of fairings and their installation. According to the results of the work conclusions.
\end{abstract}

\section{Introduction}

The construction of bridge structures occupies one of the leading positions in the construction industry: unique structures are built around the world with high speed, striking with technical solutions [1]. It is important to emphasize that the infrastructure objects being built today surprise with the refinement of architectural thought, the scale and variety of the structures used, as well as the size of the overlapped spaces. A significant role in this issue is played by technological and scientific progress [1,2]. It is thanks to the two jointly operating industries today that there are a number of opportunities that allow you to create unique objects from both aesthetic and technical points of view.

\section{Figures and tables}

Designing bridge structures is a multicomponent task: when developing documentation for such an object, it is necessary to foresee a number of factors of technogenic and natural origin. It is important to emphasize that bridges are flexible structures, which complicates the project development process at each stage several times [3].

It should be noted that today special attention is paid to the study of the effects of various natural influences, including the climate component. Large-scale bridge structures, which

\footnotetext{
* Corresponding author: js-995@mail.ru
} 
are flexible structures, are more susceptible to horizontal influence of the wind. This fact is not ignored in the regulatory documents:

1. In SP 46.13330.2012 "Bridges and pipes" [4] it is indicated that hanging and cablestayed, as well as steel beam bridges with spans of more than $100 \mathrm{~m}$ should be checked for aerodynamic stability and spatial rigidity. Also in this regulatory document it is noted that for structures with dynamic characteristics, studies should be conducted on models.

2. In Eurocode 1: Actions on structures. Part 1-4: General actions. (National Annexes) [5] governed the need for aerodynamic testing.

3. American Society of Civil Engineers. Minimum design loads for buildings and other structures [6]. ANSI / ASCE 7-98 establishes the need for testing bridge structures in wind tunnels.

The influence of wind on the strength and reliability of flexible structures is very large. The following aeroelastic reactions may appear as a result of the effect of the air flow on the bridge structures: the flutter phenomenon, galloping, vortex excitation, buffering, and parametric resonance [7]. These phenomena are caused by the dynamic effects of wind. It should be noted that each of the listed structural reactions represents a great danger to people $[3,7]$.

Resonance in the design of bridge structures was caused by the destruction of the Takom Bridge in 1940 (suspension bridge in Washington State / USA): during the occurrence of dynamic torsional vibrations (the phenomenon of aeroelastic flutter) were cut off from the design of the suspension bridge of the central span, which caused the lateral spans and tilt pylons (Fig. 1). This incident has left a significant mark in the history of science and technology [8].

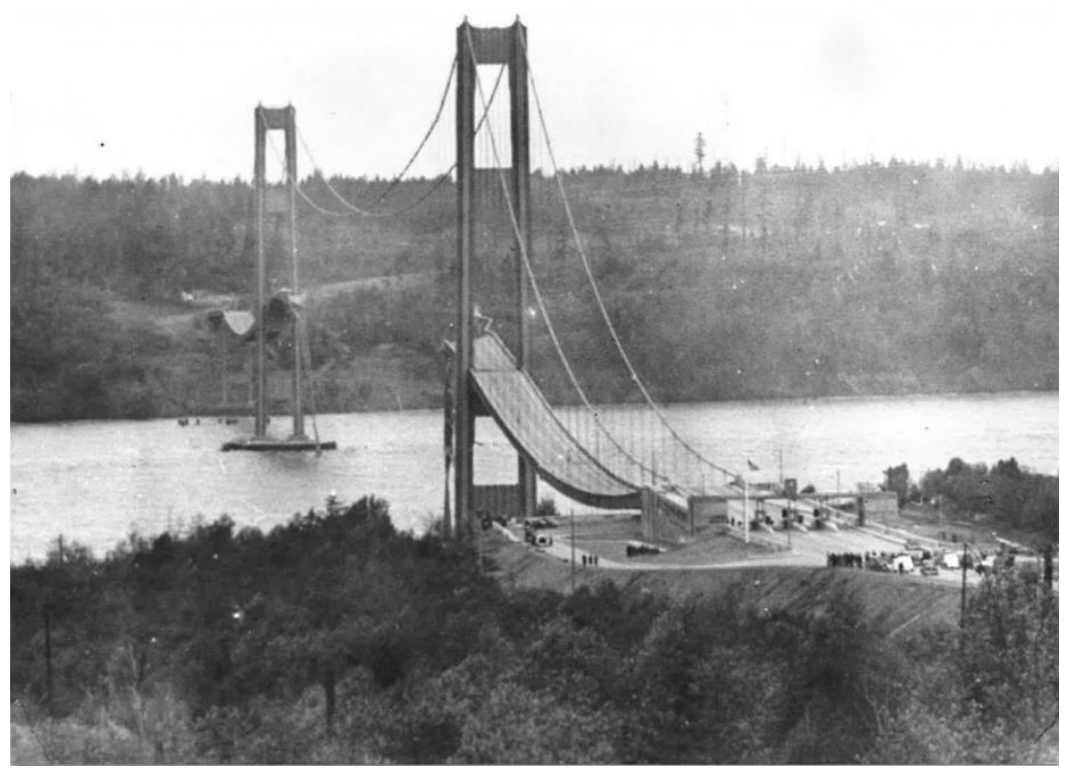

Fig. 1. Destruction of the Takom Bridge.

Another case, which attracted the attention of the scientific world again, happened in Russia (Volgograd) in 2010: an automobile bridge across the Volga River due to a significant wind load, the bridge entered into resonance with an oscillation amplitude in the vertical plane (Fig.2). In this regard, it was possible to clearly see the vibrations of structures that reached $0.5 \mathrm{~m}[9-11]$. 


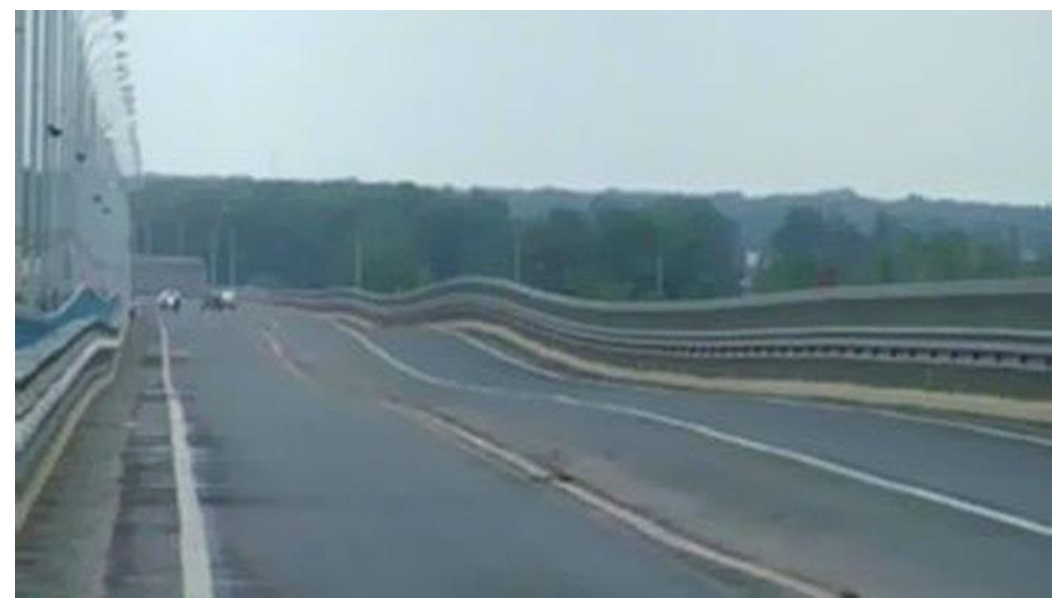

Fig. 2. Destruction of the Volgograd Bridge.

The solution to the problem of the Volgograd bridge in particular, and generally applicable to all long-span flexible structures, is the installation of damping or anti-vibration installations - dampers or fairings. It was suggested that the fairings that are widely used in aircraft manufacturing during modernization can also be applied in construction. Using one or another version of the fairing reduces the effect of wind by an order of magnitude [9, $11,12]$.

\section{Studies of wind effects on bridge structures}

Let us consider the application of the method for studying wind effects on bridge structures developed by the staff of UNPL AAISK NRU MGSU, on the example of a large-span bridge under construction in Moscow. The object under study is a beam bridge with a length of central span of $206.5 \mathrm{~m}$. The height of the rigidity beam from the water surface is $27.8 \mathrm{~m}$ (Fig.3).

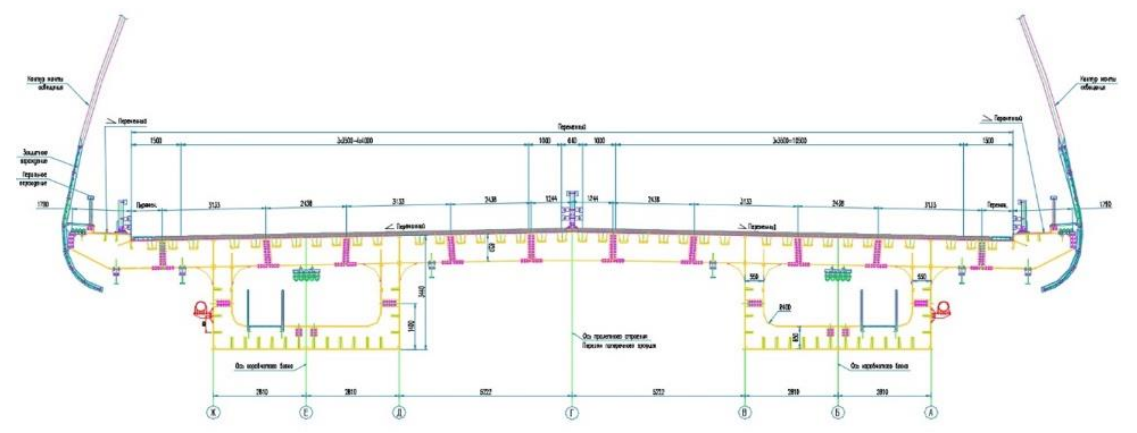

Fig. 3. The object under study.

Based on the analysis of the initial data, it was found that on the first bending and first torsional vibration modes, the largest amplitudes are observed precisely on the central span. High vibration amplitudes at the extreme spans occur at higher frequencies (Fig.4).

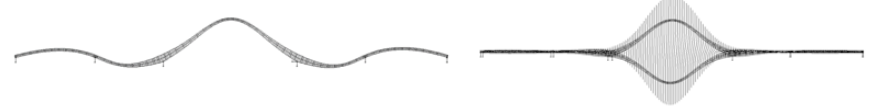

Fig. 4. Oscillation amplitudes. 
In this case, it was found that to evaluate the aerodynamic stability is sufficient to conduct tests of the sectional model of the central span.

Currently, the international community of wind energy and industrial aerodynamics (International Associations for Wind Engineering) has officially adopted two methods for studying wind effects on bridge structures:

1. Tests of sectional (cut-off) models of spans in specialized stands;

2. Testing of scale models of the entire bridge structure.

The most common method of research is testing sectional models, this method is advisable to use for beam bridges, in the absence of high-altitude pylons and the need to take into account the impact of the cable system on the superstructure. This is due to the complexity of accurate reproduction of all frequency and mass-inertial characteristics of the bridge structure in the manufacture of a scale model, with a significant decrease in the scale of the model, and, accordingly, the difficulty of interpreting the results.

When testing a sectional model, a section of the longest span of the bridge is simulated, as it is the most susceptible to dynamic wind effects. The model is made as rigid as possible, the dynamic characteristics are reproduced by using spring suspensions in specialized stands. Separate requirements for testing sectional models are given in ODM 218.2.0402014 "Guidelines for the evaluation of the aerodynamic characteristics of sections of bridge span structures" [13].

The phenomenon of bridge instability, which is caused by wind, usually involves vertical (bending) and torsional vibrations of the structure. The experimental method used to study and prevent these effects relies on a section model, which is part of the bridge span, suspended on a spring device to simulate bending and torsional vibrations with the imposition of both forms of vibration, which is exposed to wind in a wind tunnel [14].

When conducting dynamic tests, the model is fixed on spring hangers in a specialized stand, at a slip angle of $0^{\circ}$, as the most unfavorable from the point of view of aerodynamics. The characteristics of the spring suspensions and the corresponding scale of the flow velocity are determined at the design stage of the model. The level of damping of the model is measured and is 0.02 , which meets the requirements of domestic and foreign regulatory documents, the low value of the level of damping is provided by a rigid metal structure of the layout and its low dissipative properties. Model movements are measured using 4 optical sensors that operate on the principle of laser beam triangulation. The measurement does not affect the free movement of the suspended model; bending and torsion are measured in the same way, by adding or reducing the number of sensor signals. Wind speed is measured using a Pitot tube connected to a highly sensitive pressure sensor. All motion sensor signals first pass a low-pass filter, then digitized using a 16-bit analog-todigital converter at a speed of 1000 values per second. Statistical calculation of the recorded data allows to obtain average values, root-mean-square, maximum, minimum, etc. for all measurement channels. Measurement of movement is carried out relative to the zero level at a wind speed of $0 \mathrm{~m} / \mathrm{s}[14,15]$.

Measurement of sustainability is presented as a graph of displacement in full scale versus average wind speed also in full scale. Separate graphs are made for two variants of the protective shield design - continuous and permeable (Fig.5). 


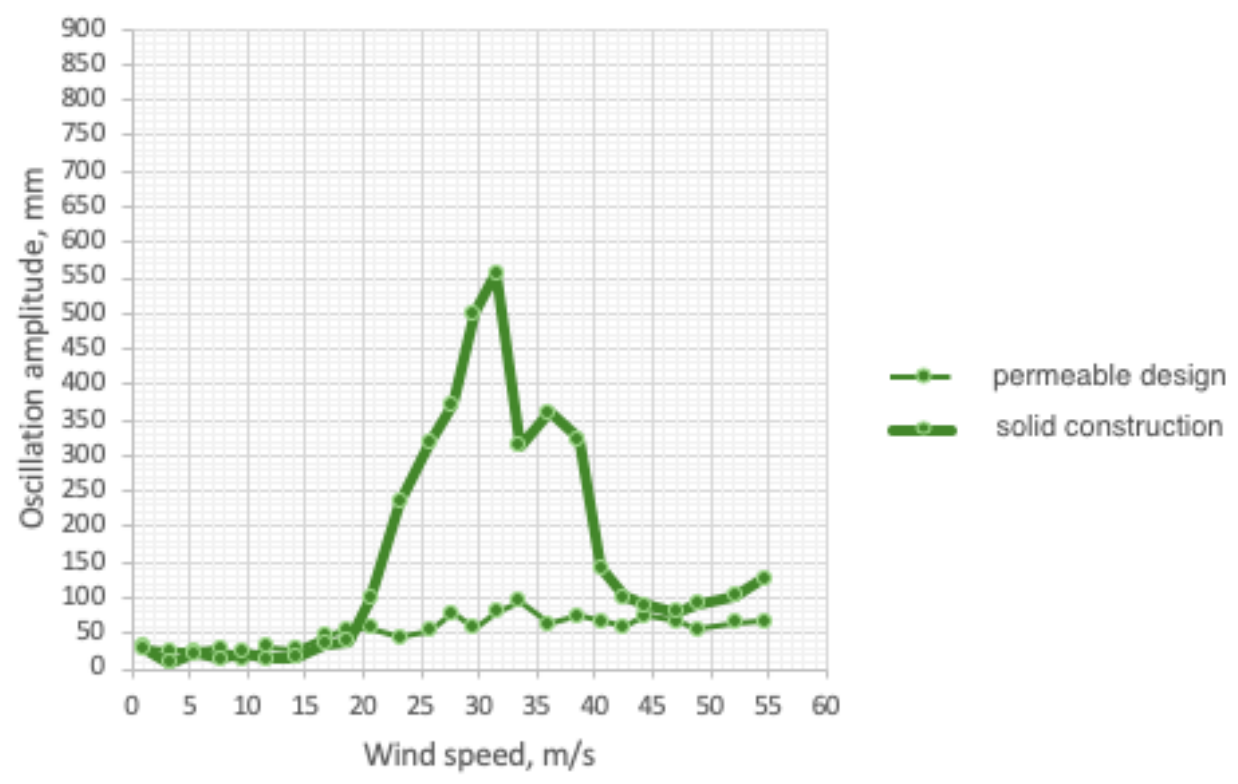

Fig. 5. Dependence of the amplitude of oscillations of the bridge span on the wind speed, flow direction $\alpha=0^{\circ}$.

Thus, the dynamic tests of the model showed that the permeable design of the protective screen is preferable from the point of view of the aerodynamics of the superstructure. Nevertheless, according to the results of an experimental study of the stability of a span with a permeable protective shield, a vortex phenomenon was also detected; excitations at the following wind speeds: with a flow direction of 0 with a flow velocity of 47 to $64 \mathrm{~m} / \mathrm{s}$ with an increase in the amplitude of oscillation up to $300 \mathrm{~mm}$ with a frequency of oscillation of $1.33 \mathrm{~Hz}$ [14].

In order to increase the aerodynamic stability of the design, the Customer is offered to use the aerodynamic fairing according to the sketch (Fig.6).

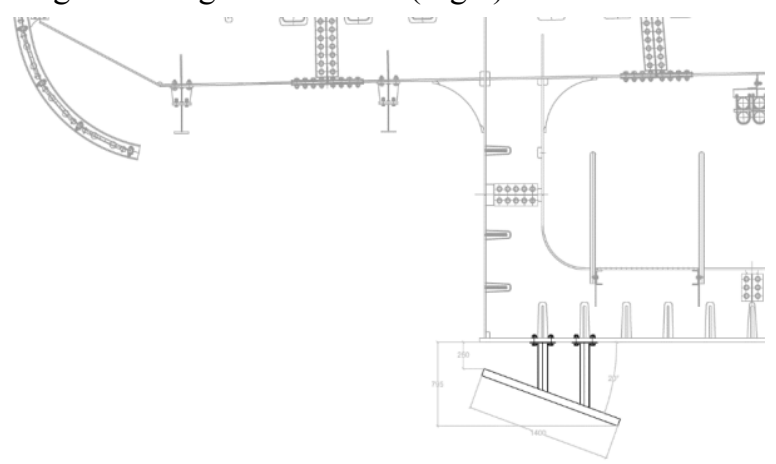

Fig. 6. Sketch of fairing.

According to the results of an additional experimental study of stability, the phenomenon of vortex excitation was revealed at the following wind speeds: with a flow direction of 0 degree with a flow velocity from 47 to $64 \mathrm{~m} / \mathrm{s}$ with an increase in the amplitude of oscillations up to $113 \mathrm{~mm}$ with a frequency of vibrations of $1.33 \mathrm{~Hz}$. With the use of an 
aerodynamic fairing, the stability of the span structure as a whole increases, the amplitude of oscillations with the flow direction of 0has decreased by more than 2 times (Fig.7).

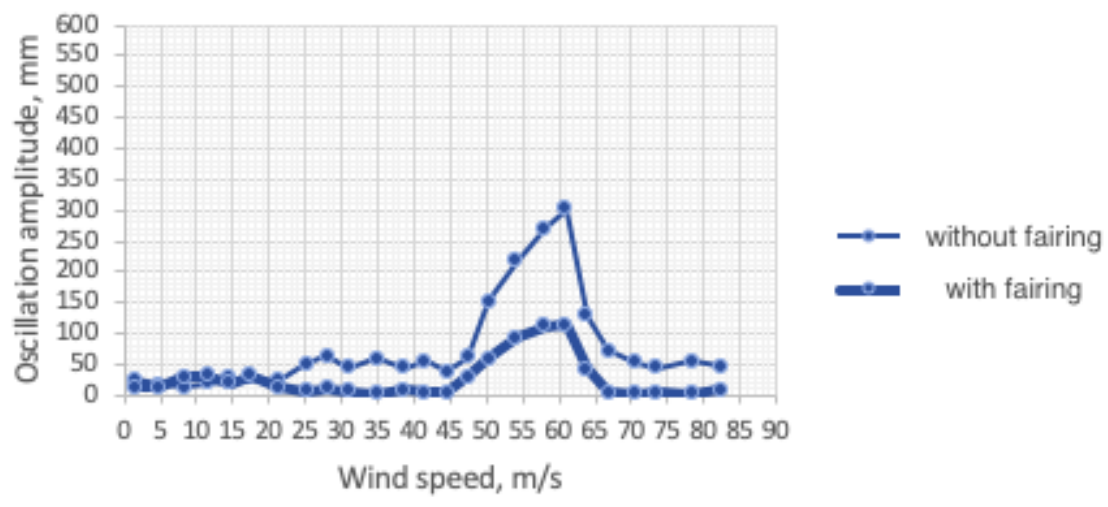

Fig. 7. Dependence of the amplitude of oscillations of the bridge span on the wind speed, flow direction $\alpha=0^{\circ}$.

\section{Conclusion}

The choice of the optimal cross-sectional shape and the assessment of the stability of the bridge structure is a complex engineering task [11, 12]. The best solution now is experimental research in wind tunnels, in conjunction with the analytical calculations used in the processing of experimental results. The use of modern measuring systems and test benches make it possible to minimize the possibility of damage and destruction of the structure. The results presented in the article show a significant influence of aerodynamic fairings, as well as permeability of protective screens on the stability of the structure, which tells us about the need to participate in the tests not only specialists in the direction of aerodynamics, but also designers and architects. Conducting such studies is now an integral part of the design of long-span bridge structures directly related to ensuring the safety of these structures $[4-6,11,14,15]$.

This work was financially supported by the Ministry of Education and Science (state task \#7.6075.2017/Bch). All tests were carried out using research equipment of The Head Regional Shared Research Facilities and Unique Scientific Installation Large Research Gradient Wind Tunnel of the Moscow State University of Civil Engineering.

\section{References}

1. I.G. Ovchinnikov, Nauka: 21 vek, 113 (2011)

2. Y. Yang First Published (2017)

3. D. Romanic Journal of Wind Engineering and Industrial Aerodynamics 184 (2019)

4. SP 46.13330.2012 Bridges and pipes

5. Eurocode 1: Actions on structures. Part 1-4: General actions. (National Annexes)

6. American Society of Civil Engineers. Minimum design loads for buildings and other structures.

7. A. Larsen, Journal of Sound and Vibration, 3346 (2015)

8. K. Yusuf, Billah American Journal of Physics, 59118 (1991) 
9. I.I. Ovchinnikov, I.G. Ovchinnikov, V.O. Filippov, Naukovedenie, 76 (2015)

10. U.L. Rutman, V.A. Meleshko, Stroitelnaya mehanika i raschet sooruzheniy 3 (2011)

11. S.K. Pshenichnikov, Vestnik mostostroeniya 1 (2011)

12. G.A. Naumova, S.A. Ponomarenko, Vestnik grazhdanskikh inzhenerov 356 (2012)

13. ODM 218.2.040-2014 Guidelines for the evaluation of the aerodynamic characteristics of sections of bridge span structures

14. P.S. Churin, Nauchnoe obozrenie 8 (2015)

15. O.O. Egorychev, O.I. Poddaeva, P.S. Churin, Nauchnoe obozrenie 9 (2015) 